\title{
A Boy with an LCR3/4-Flanked 10q22.3q23.2 Microdeletion and Uncommon Phenotypic Features
}

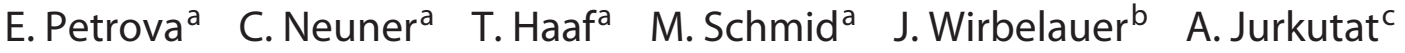 \\ K. Wermke ${ }^{d} \quad$ I. Nanda ${ }^{a} \quad$ E. Kunstmann ${ }^{a}$ \\ anstitute for Human Genetics, b University Children's Hospital, ' Department of Special Education, Speech and \\ Language Pathology, and d Department of Orthodontics, Center for Pre-Speech Development and Developmental \\ Disorders, University of Wuerzburg, Wuerzburg, Germany
}

\section{Key Words}

BMPRA 1 - Cleft palate - Language development .

LCR3/4-flanked 10q22.3q23.2 deletion

\begin{abstract}
The recurrent $10 \mathrm{q} 22.3 \mathrm{q} 23.2$ deletion with breakpoints within low copy repeats 3 and 4 is a rare genomic disorder, reported in only 13 patients to date. The phenotype is rather uncharacteristic, which makes a clinical diagnosis difficult. A phenotypic feature described in almost all patients is a delay in speech development, albeit systematic studies are still pending. In this study, we report on a boy with an LCR3/4flanked 10q22.3q23.2 deletion exhibiting an age-appropriate language development evaluated by a standardized test at an age of 2 years and 3 months. The boy was born with a cleft palate - a feature not present in any of the patients described before. Previously reported cases are reviewed, and the role of the BMPR1A gene is discussed. The phenotype of patients with an LCR3/4-flanked 10q22.3q23.2 deletion can be rather variable, so counseling the families regarding the prognosis of an affected child should be done with caution. Long-term studies of affected children are needed to delineate the natural history of this rare disorder.
\end{abstract}

(c) 2013 S. Karger AG, Basel
(C) 2013 S. Karger AG, Base

1661-8769/13/0051-0019\$38.00/0
Balciuniene et al. [2007] reported 2 patients with a heterozygous 10q22q23 deletion flanked by low copy repeats (LCRs), designated LCR3 and LCR4. Low copy repeats are known to be hotspots for genomic rearrangements. Since this first report, 13 further cases of such recurrent deletions with breakpoints within LCR3 and LCR4 have been published [Alliman et al., 2010; Reddy et al., 2011; Singh et al., 2011; van Bon et al., 2011]; these are summarized in table 1.

LCR3/4-flanked 10q22.3q23.2 deletions present a recurrent genomic disorder with a well-defined genotype, deleted segments ranging from 7.2 to $7.5 \mathrm{Mbp}$ in size. The common phenotype of the reported patients with such deletions included facial dysmorphic features, such as hypertelorism, up- or downslanting palpebral fissures and flat nasal bridge, developmental delay of varying degrees most notable in language acquisition as well as congenital heart defects (CHD), high-arched palate and club feet in some of the patients. Some of the genes within the deleted region, e.g. NRG3, GRID1, BMPR1A, GLUD1, have been discussed as putative candidate genes associated with the phenotype, especially regarding the neuropsychological development [Balciuniene et al., 2007; van Bon et al., 2011]. In this paper, we focus on the role of $B M P R 1 A$ (bone morphogenetic protein receptor, type

\section{KARGER}

E-Mail karger@karger.com

www.karger.com/msy
Erdmute Kunstmann

Department of Human Genetics

University of Wuerzburg

Am Hubland, DE-97074 Wuerzburg (Germany)

E-Mail kunstmann@biozentrum.uni-wuerzburg.de 


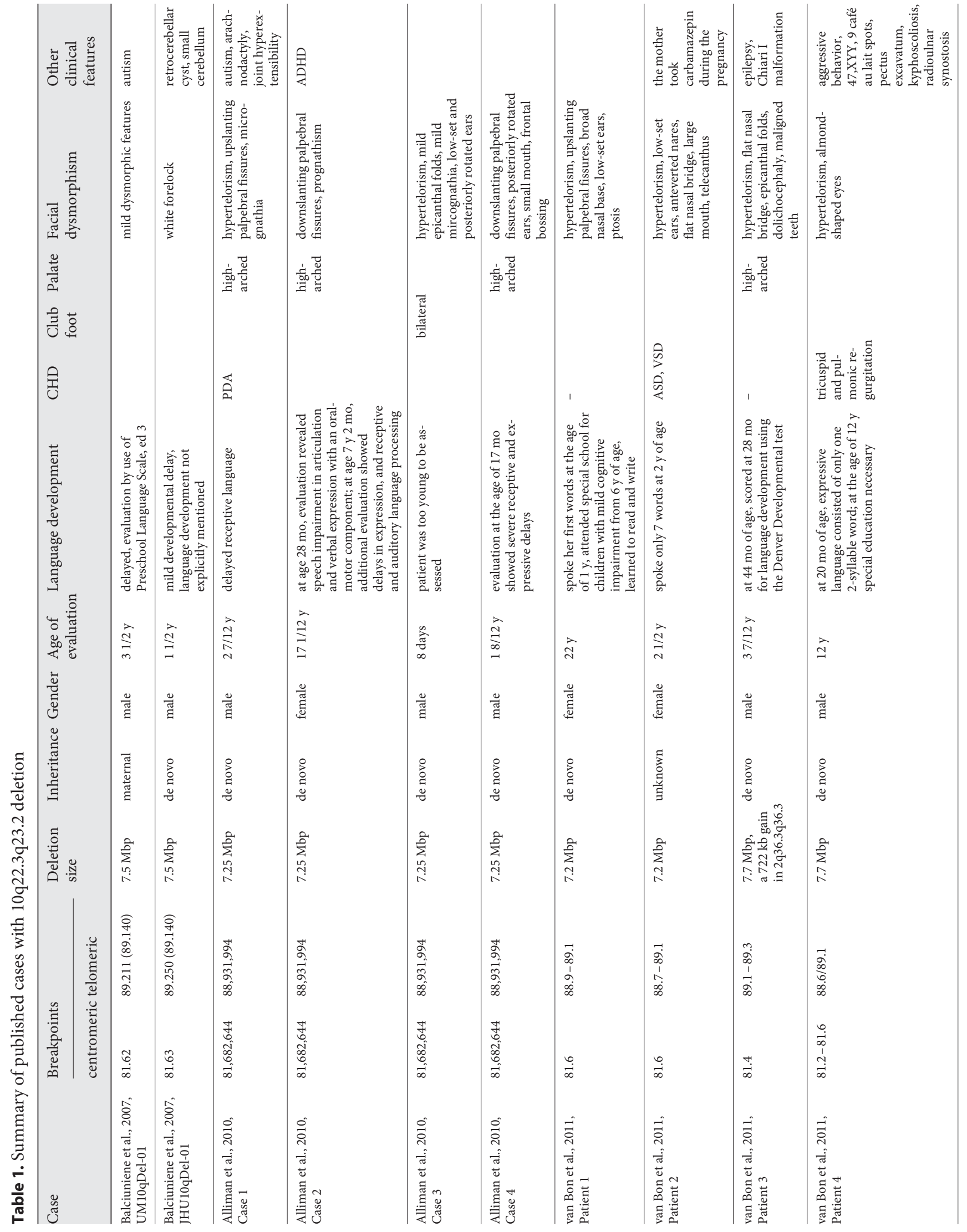




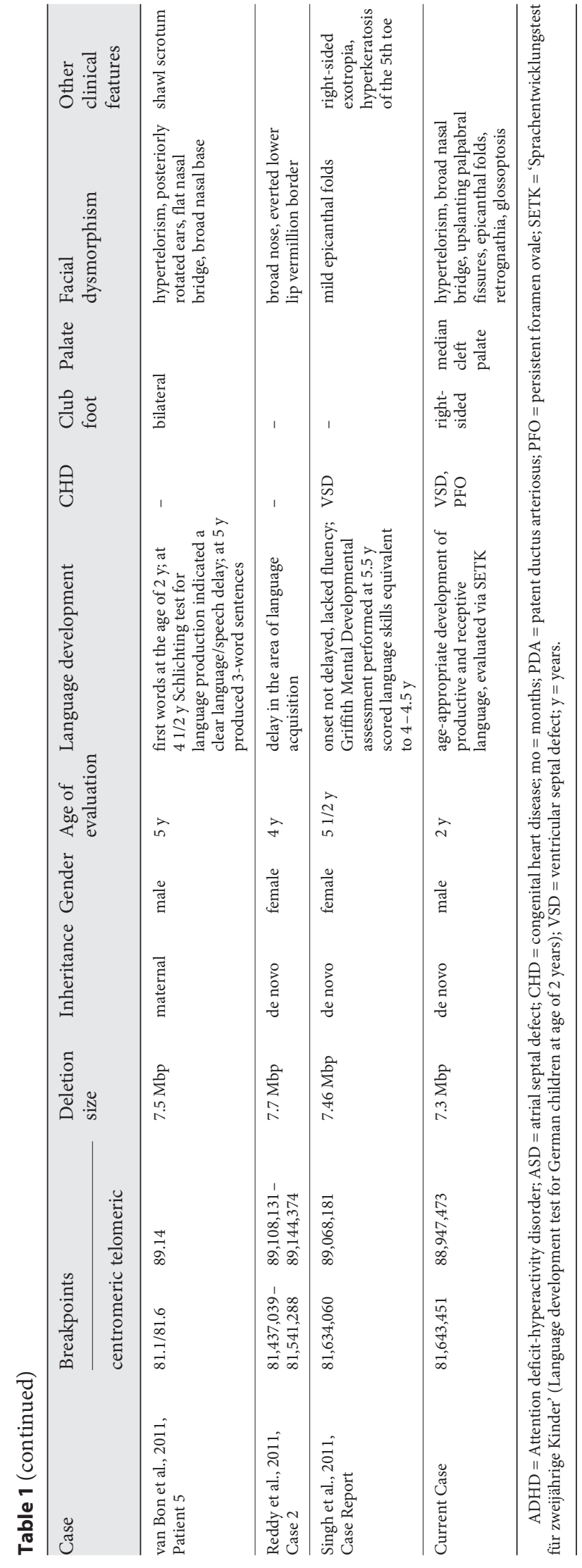

1A). BMPR1A encodes for a transmembrane serine/threonine kinase which acts as a receptor for ligands of the BMP (bone morphogenetic proteins) family of growth factors. BMP signaling plays an important role in human embryonic development, especially in the formation of bone, cartilage, neural and heart tissues [Chen et al., 2004]. Heterozygous mutations of $B M P R 1 A$ are known to cause juvenile polyposis syndrome (JPS, OMIM 174900), and there is also some evidence that BMPR1A mutations may underlie CHD and craniofacial dysmorphism.

Here we report on a boy with an LCR3/4-flanked 10q22.3q23.2 deletion, who did not show any deficits in his language development. However, he is the first reported patient with a cleft palate which seriously affects his speech intelligibility. His speech is characterized by articulation errors frequently heard in the speech of individuals with a cleft palate prior to 3 years of age [GoldingKushner, 2001].

\section{Clinical Report}

This male patient was $23 / 12$ years old at the time of genetic evaluation. He was born after $40+1$ weeks of gestation via spontaneous vaginal delivery after an uneventful pregnancy. Weight, height and head circumference at birth were $2,990 \mathrm{~g}, 48.5 \mathrm{~cm}$ and $34 \mathrm{~cm}$, respectively. A right-sided club foot, a cleft palate and facial dysmorphic features, including broad nasal bridge, high forehead, upslanting palpebral fissures, epicanthal folds, and retrognathia were noticed after birth. Because of the low $\mathrm{O}_{2}$ saturation, the boy was transferred to the intensive care unit immediately after birth. Echocardiographic evaluation showed a small, hemodynamically irrelevant ventricular septum defect (VSD) and persistent foramen ovale.

The boy showed recurrent $\mathrm{O}_{2}$ saturation falls due to retrognathia and glossoptosis; therefore, home-monitoring was provided. Three days after birth, a special palatal plate was inserted (orthodontic plate therapy) [for details, see Kochel et al., 2011]. At the age of $12 / 12$ years, surgical closure of the cleft palate was performed. The boy had surgical correction of the club foot at the age of 6 months after an unsuccessful nonsurgical treatment.

By the age of 2 years the VSD and persistent foramen ovale were no longer echocardiographically detectable. The patient showed normal motor development, but at the age of 26 months, his height was $80.5 \mathrm{~cm}$ ( $2 \mathrm{~cm}$ below 3rd percentile) and body weight was $10,900 \mathrm{~g}$ (3rd percentile). Head circumference was normal. The boy's language outcome was assessed using a standardized test for German children developed to measure both receptive and expressive language skills in 24-35-month-old children (SETK-2) [Grimm, 2000]. It consists of 4 subtests with different items assessing performances in the production and comprehension of words and sentences via direct examination of the children by trained speech and language therapists. He successfully performed all 4 subtests.

The patient's parents (mother 30 years old, father 32 years old at the child's birth) were healthy, nonconsanguineous Caucasians. A pregnancy 7 years earlier ended with spontaneous abortion after 9 weeks of gestation. The further family history was unremarkable. 


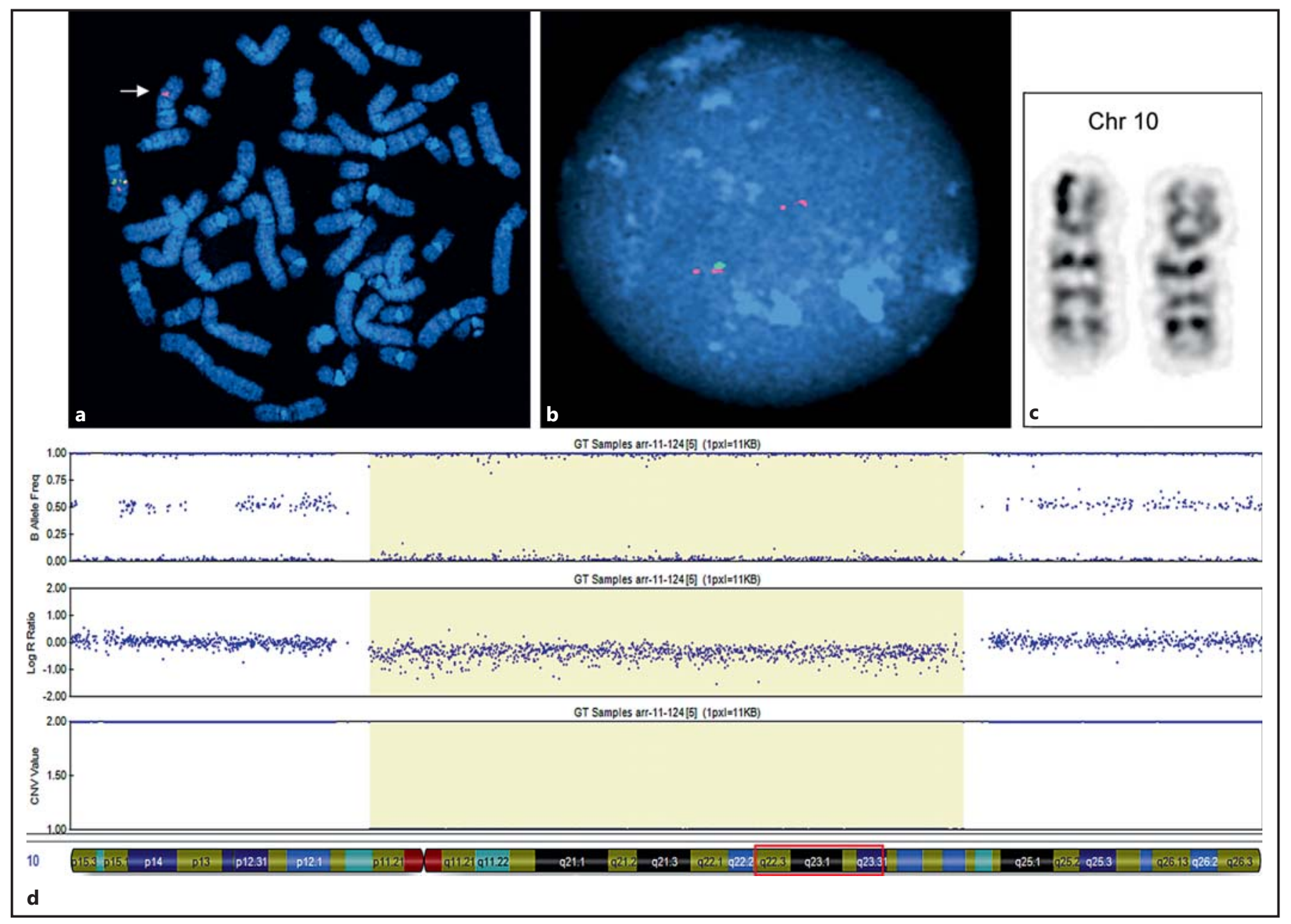

Fig. 1. FISH results of the proband. a Simultaneous hybridization of BAC probes RP11-479O17 (green) and RP11-322M19 (red) to metaphase spread validating a 10q22.310q23.2 deletion on one homolog of chromosome 10 (arrow), as the deleted chromosome displays no hybridization signal for the probe RP11-479O17 (green) representing the deleted region, whereas the hybridization signal of flanking probe RP11-322M19 (red) is marked on both homologs. $\mathbf{b}$ Hybridization of the same BACs on an interphase nucleus showing a single hybridization signal of RP11-479O17 (green) closely associated to the flanking probe, RP11-322M19 (red). Note

\section{Genetic Diagnostics}

The GTG-bands of the proband's chromosomes showed a normal male karyotype 46,XY. Upon this a SNP array analysis was performed, which revealed a 7,304-kb deletion on the long arm of chromosome 10, at 10q22.3q23.2 [ $\operatorname{arr}($ hg19)10q22.3q23.2 $(81,643,451-88,947,473) 81]$ with breakpoints within LCR3 and LCR4 [see Balciuniene et al., 2007 for a list of the deleted genes]. The deleted chromosome 10 was verified by FISH analysis with informative BAC probes (fig. 1). More than 100 interphase nuclei and additional 23 metaphases were analyzed in the deleted region. The the split signal of the flanking probe, RP11-322M19 (red) is due to the initiation of replication of the region. c GTG-bands of chromosome 10 in the proband illustrating no pronounced difference in the banding pattern between the homologs. d Human CytoSNP12 array analysis in the proband shows a $7.3-\mathrm{Mb}$ deletion in $10 \mathrm{q} 22.310 \mathrm{q} 23.2$ shown in yellow. The uninterrupted deletion in the region is marked through an absence of heterozygote alleles in $B$ allele frequency (top), a reduced intensity (below normal range) of markers in log $\mathrm{R}$ ratio (middle) and the CNV value plot (bottom) indicating a copy number of one. 


\section{Methods}

\section{Cytogenetics}

Chromosomes were prepared from PHA stimulated peripheral blood lymphocytes of the index patient as well as the parents following a standard procedure, and standard karyotyping was performed based on GTG-banding at a level of approximately 450 bands.

\section{Molecular Karyotyping}

In order to identify cryptic chromosomal changes, a genomewide SNP array was performed using the HumanCytoSNP-12v2.1 BeadChip Kit (Illumina Inc., San Diego, Calif., USA). This array contains 300,000 markers distributed with an average interSNP distance of around $10 \mathrm{~kb}$. Briefly, genomic DNA was prepared from peripheral blood following the standard salt extraction method, and 200 ng of genomic DNA from the index patient and parent were hybridized to the BeadChip in an Infinium ${ }^{\circledR}$ HD Ultra Assay according to the manufacturer's protocol. After hybridization, the BeadChip was scanned with the Illumina BeadArray Reader, and the data were analyzed by examining signal intensity (log $\mathrm{R}$ ratio) and allelic composition (BAF) with GenomeStudio v2010.1 and cnvPartition v3.1.6 software. A minimum of a 5-probe cut-off value was used to define a copy number change. The call rates of the samples were larger than $99.0 \%$.

\section{FISH Analysis}

BAC clones from the 10q22.3q23.2 region were selected from the Ensembl genome browser site (http://www.ensembl.org/) and ordered from the Children's Hospital Oakland Research Institute (http://bacpac.chori.org/). DNA was extracted by alkaline lysis and labeled by nick translation with Fluorescein-12-dUTP (Roche) or Tetramethyl-Rhodamine-5-dUTP (Roche). After hybridization, washing and counterstaining chromosomes were analyzed with a Zeiss AxioImager microscope. Image acquisition and analysis were performed using a CCD camera and FISHView 2.0 software (Applied Spectral Imaging). At least 20 metaphases and additional 100 interphase nuclei were evaluated per BAC probe.

\section{Discussion}

One phenotypic feature present in almost all of the published cases of LCR3/4-flanked 10q22.3q23.2 deletion that have been published is a developmental delay of varying degrees, most prominently in language and speech. However, the latter claim must be put into perspective with the fact that the majority of patients in previous studies were not evaluated by standardized tests. Most available information seems to reflect parental attitudes or occasional clinical evaluation. The current patient was examined by a standardized language test, and in contrast to other patients, he showed a normal development of receptive and expressive language at the age of 2 years. Thus, this is a rare case of a patient with an LCR3/4-flanked 10q22.3q23.2 deletion, who shows no speech/language impairment upon objective evaluation. In view of the considerable variance in speech/language delay ranging from mild to severe in the majority of published patients (table 1), the normal development of language acquisition and production of of the index patient may be the result of an even broader phenotypic expressivity of the underlying chromosomal microdeletion. Otherwise, it is possible that the test was not sensitive enough to detect a mild deficit or that speech/language delay may manifest at a later age. Therefore, further examinations with standardized language tests are planned at the age of 4 and 5 years.

Another phenotypic feature present in all patients is craniofacial dysmorphism, most commonly hypertelorism $(8 / 14,57 \%)$, flat/broad nasal bridge $(6 / 14,43 \%)$, upslanting or downslanting palpebral fissures (5/14, $36 \%)$, and high-arched palate $(4 / 14,29 \%)$. Other rare features are micrognathia, low-set or posteriory-rotated ears, and epicanthal folds; the patient presented here is the first one exhibiting a mediane cleft palate. In a recent publication, Saito et al. [2012] presented a conditional knockout mouse model, generated by expressing a dominant negative BMPR1A protein (dnBMPR1A) in neuralcrest-derived cells (dnBMPR1A lacks the intracellular kinase domain and, thus, inhibits the BMPR1A-mediated signaling pathway). The mutant mice exhibited either facial fusion defects such as a cleft face and cleft palate, or facial dysmorphology corresponding to hypertelorism and flat nasal bridge in humans. An incomplete expansion of neural-crest-derived mesenchymal cells due to extensive apoptosis was found in the mutant embryos. In addition, $50 \%$ of the mutant mice with a facial cleft also showed heart defects. The patient reported here also showed a small, VSD and persistent foramen ovale. Thus, the craniofacial dysmorphic features and the heart septal defects observed in the patients with LCR3/4-flanked 10q22.3q23.2 deletion may be partly caused by a reduction of BMPR1A-mediated signaling.

The BMPR1A gene is also associated with the JPS that is characterized by the development of hamartomatous polyps in the gastrointestinal tract. Heterozygous point mutations or partial deletions of BMPR1A are found in approximately $20 \%$ of patient with JPS [Larsen et al., 2011]. Among patients with 10q22q23 microdeletions, JPS has been observed only in those patients having both genes BMPR1A and PTEN deleted. PTEN is a gene associated with Cowden syndrome and Bannayan-Riley-Ruvalcaba syndrome involved in the deletion, suggesting that the contiguous deletion of both BMPR1A and PTEN is required in order for polyposis to manifest [Delnatte et 
al., 2006; Dahdaleh et al., 2012]. PTEN is telomeric to $B M P R 1 A$ as well as to LCR4, so it is not involved in the LCR3/4-flanked 10q22.3q23.2 deletion; none of the patients with such deletions were reported to have JPS, supporting the supposition that the contiguous deletion of both BMPR1A and PTEN is needed for the development of the polyposis phenotype. Unfortunately, the number of described patients is very small, and in rare cases, patients with mutations in BMPR1A showed Bannayan-Riley-Ruvalcaba syndrome-like features [Zhou et al., 2001]. Therefore, an accurate genotype-phenotype correlation is not possible. In patients with JPS, the risk of gastrointestinal cancer is increased even though the majority of polyps are benign. Our patient did not show any JPS symptoms at the time of evaluation. Nevertheless, he was considered as a patient of risk for JPS, and baseline screening according to Larsen et al. [2011], including a complete blood count, colonoscopy and upper gastrointestinal endoscopy beginning at 15 years of age or at initial symptoms such as gastrointestinal bleeding was recommended.

Five of the 14 patients (36\%), including the one presented here, had CHD, most commonly VSD. Breckpot et al. [2012] published a case report on a boy with VSD, short stature and facial dysmorphism, who had an intragenic BMPR1A deletion. Reviewing literature for cases with distal chromosome $10 \mathrm{q}$ deletions and using computed gene prioritization, the authors showed that $B M P R 1 A$ is the best candidate gene for CHD in patients with 10q22q23 deletions. Saito et al. [2012] also evaluated heart morphology of the mutant animals and showed that some of those with a facial cleft exhibited a ventricular septum defect as well. Thus, deletions of BMPR1A may contribute to various phenotypes. Detailed phenotypic characterization of patients with $B M P R 1 A$ deletions and other mutations is warranted, to further delineate the role of BMPR1A in the clinical presentation of the LCR3/4flanked 10q22.1q23.2 deletion syndrome and in human embryonic development in general.

In conclusion, the phenotype of the LCR3/4-flanked 10q22.1q23.2 deletion varies significantly, especially in speech and language development, ranging from severe delay to age-appropriate development as in the patient presented here. Thus, the prognosis of infants with an ascertained genotype regarding their language development should be given with caution. Long-term follow-up studies of affected patients are needed to further delineate the natural history of this rare disorder.

\section{Acknowledgement}

The authors thank the family described in this clinical report for participating in the study.

\section{References}

-Alliman S, Coppinger J, Marcadier J, Thiese H, Brock $\mathrm{P}$, et al: Clinical and molecular characterization of individuals with recurrent genomic disorder at 10q22.3q23.2. Clin Genet 78:162-168 (2010).

Balciuniene J, Feng N, Iyadurai K, Hirsch B, Charnas L, et al: Recurrent 10q22-q23 deletions: a genomic disorder on $10 \mathrm{q}$ associated with cognitive and behavioral abnormalities. Am J Hum Genet 80:938-947 (2007).

- Breckpot J, Tranchevent LC, Thienpont B, Bauters M, Troost E, et al: BMPR1A is a candidate gene for congenital heart defects associated with the recurrent 10q22q23 deletion syndrome. Eur J Med Genet 55:12-16 (2012).

Chen D, Zhao M, Mundy G: Bone morphogenetic proteins. Growth Factors 22:233-241 (2004).

Dahdaleh FS, Carr JC, Calva D, Howe JR: Juvenile polyposis and other intestinal polyposis syndromes with microdeletions of chromosome 10q22-23. Clin Genet 81:110-116 (2012).

-Delnatte C, Sanlaville D, Mougenot JF, Vermeesch JR, Houdayer C, et al: Contiguous gene deletion within chromosome arm 10q is associated with juvenile polyposis of infancy, reflecting cooperation between the $B M P R 1 A$ and PTEN tumor-suppressor genes. Am J Hum Genet 78:1066-1074 (2006).

Golding-Kushner KJ: Therapy Techniques for Cleft Palate Speech and Related Disorders (Singular Publishing, San Diego 2001).

Grimm H,Aktas M, FrevertS: SETK-2. Sprachentwicklungstest für zweijährige Kinder. Diagnose rezeptiver und produktiver Sprachverarbeitungsfähigkeiten. Hogrefe-Verlag für Psychologie, Göttingen, 2002.

Kochel J, Meyer-Marcotty P, Wirbelauer J, Böhm $\mathrm{H}$, Kochel M, et al: Treatment modalities of infants with upper airway obstruction-review of the literature and presentation of novel orthopedic appliances. Cleft Palate Craniofac J 48:44-55 (2011).

Larsen Haidle J, Howe JR: Juvenile Polyposis Syndrome, in Pagon RA, Bird TD, Dolan CR, et al. (eds): GeneReviews ${ }^{\mathrm{TM}}$ (University of Washington, Seattle 1993). http://www.ncbi. nlm.nih.gov/books/NBK1469/.

Nowakowska BA, de Leeuw N, Ruivenkamp CA, Sikkema-Raddatz B, Crolla JA, et al: Parental insertional balanced translocations are an important cause of apparently de novo CNVs in patients with developmental anomalies. Eur J Hum Genet 20:166-170 (2012).

Reddy KS, Mardach R, Bass H: Oligoarray (105K) CGH analysis of chromosome microdeletions within 10q22.1q24.32. Cytogenet Genome Res 132:113-120 (2011).

- Saito H, Yamamura KI, Suzuki N: Reduced bone morphogenetic protein receptor type $1 \mathrm{~A}$ signaling in neural-crest-derived cells causes facial dysmorphism. Dis Model Mech 5:948955 (2012).

Singh S, Aftimos S, George A, Love DR: Interstitial deletion of 10q23.1 and confirmation of three 10qdel syndromes. Singapore Med J 52:e143-146 (2011).

van Bon BW, Balciuniene J, Fruhman G, Nagamani SC, Broome DL, et al: The phenotype of recurrent 10q22q23 deletions and duplications. Eur J Hum Genet 19:400-408 (2011).

Zhou XP, Woodford-Richens K, Lehtonen R, Kurose $\mathrm{K}$, Aldred $\mathrm{M}$, et al: Germline mutations in BMPR1A/ALK3 cause a subset of cases of juvenile polyposis syndrome and of Cowden and Bannayan-Riley-Ruvalcaba syndromes. Am J Hum Genet 69:704-711 (2001). 